\title{
Erratum: Field Cancerization in Sporadic Colon Cancer
}

Soo-Kyung Park ${ }^{1,2}$, Chang Seok Song ${ }^{1,2}$, Hyo-Joon Yang ${ }^{1,2}$, Yoon Suk Jung ${ }^{1,2}$, Kyu Yong Choi ${ }^{1,2}$, Dong Hoe Koo ${ }^{1,2}$, Kyung Eun $\mathrm{Kim}^{2,3}$, Kyung Uk Jeong ${ }^{2,4}$, Hyung Ook Kim ${ }^{2,4}$, Hungdai Kim ${ }^{2,4}$, Ho-Kyung Chun ${ }^{2,4}$, and Dong II Park ${ }^{1,2}$

${ }^{1}$ Division of Gastroentorology, Department of Internal Medicine, ${ }^{2}$ Gastrointestinal Cancer Center, Departments of ${ }^{3}$ Pathology, and ${ }^{4}$ Surgery, Kangbuk Samsung Hospital, Sungkyunkwan University School of Medicine, Seoul, Korea

https://doi.org/10.5009/gnl15334

Gut Liver 2016;10(5):773-780.

In the version of this article initially published, the first affiliation (affiliation number 1) was incorrectly stated as "Division of Gastroentorology, Department of Internal Medicine.” The correct affiliation is “Department of Internal Medicine." 